\title{
Development of a Remote Systems and Controls Laboratory
}

\author{
G.G. Parker, M.J. Agostini, M.N. Devarakonda and P.F. Zenner \\ Department of Mechanical Engineering-Engineering Mechanics (MEEM) \\ Michigan Technological University (MTU)
}

\begin{abstract}
The Department of Mechanical Engineering-Engineering Mechanics at Michigan Technological University has developed a remote version of a required undergraduate laboratory that is a practical and relevant component of an engineer's education. The purpose is to provide a combined mechanical engineering laboratory experience that reinforces the traditional elements of a curriculum - available to everyone, anywhere. As part of a quarter to semester conversion at MTU a Dynamic Systems and Controls laboratory was integrated with the traditionally required textbook course. The goal of the lab was to illustrate some of the abstract concepts of the course using "hands-on" experience. A secondary goal of the lab was to strengthen a student's understanding of Computer Aided Control System Design (CACSD). The second phase of the development of this lab was to determine and overcome the challenges and barriers of implementing the lab remotely. This paper describes our approach to converting traditional onsite control system labs to remote versions. In addition, limitations inherent to remote experiment execution are described along with our plan for testing and assessing the ability of the remote labs to convey practical aspects of control system phenomena.
\end{abstract}

\section{Introduction}

The Department of Mechanical Engineering-Engineering Mechanics at Michigan Technological University developed a required undergraduate Dynamic Systems and Controls laboratory that is a practical and relevant component of an engineer's education. The purpose is to provide a combined laboratory experience that reinforces the traditional elements of the course. Faculty are directly involved teaching the lecture component of the course and are responsible for the creation of laboratory experiments. Graduate students work closely with these faculty members and teach the weekly labs.

The primary goal of the new lab is to remove some of the abstract nature of a traditional systems and controls course and give "hands on" learning experience. The students need to interact in real time and see the effects of making changes - they need to experiment with freedom and have a relevant experience. The other goal is to enhance the understanding of CACSD by the students. The new lab goals were then expanded to determine its adaptability and effectiveness to a remote, place bound student. 


\title{
Course Objectives
}

Successful completion of this course provides the student with several skills in control system analysis and design, including:

- Understand control system terminology with application to establishing closed-loop performance requirements in both the time domain and the frequency domain.

- Differentiate between linear and nonlinear systems.

- Apply Laplace transformation to solve linear, constant coefficient differential equations and evaluate the steady-state behavior of dynamic systems.

- Design feedback control systems for dynamic systems to satisfy time domain performance requirements.

- Apply root locus and Bode methods to design closed-loop control systems.

The goal of the laboratory component is to increase the student's understanding of control system design and the implications of feedback on a system's performance. It is not intended to be an exercise in report generation. This is covered in other aspects of the Mechanical Engineering curriculum. Solidifying time domain response characteristics such as overshoot and rise time, understanding how a system's output response eventually decreases as the frequency of the input increases, learning the catastrophic nature of instability and that theoretical unboundedness of response rarely occurs due to actuator and other physical limitations are the low-level goals of the lab component.

These concepts were taught in the course prior to the inclusion of the laboratory component. Based on student response to course evaluation questions, and anecdotal evidence in final exams it was clear that a deep understanding of these practical issues was lacking. Inclusion of the laboratory component was a response to this awareness and has improved student understanding of these concepts. The question we are now attempting to answer quantitatively is, "What level of understanding of these concepts can web-based control system laboratory exercises provide even though the students never touch the hardware?" Note that the goal of this study is not to produce an engineering solution to the problem of offering remote students a laboratory experience. This can certainly be accomplished, and with care, it can be done in a manner that provides some level of understanding of hardware concepts. In contrast the goal is to understand the differences in student understanding of hardware concepts for a particular remote laboratory scenario. Hopefully this will provide guidance for future remote laboratory development if it still appears to be a promising direction. One possible outcome is that the remote lab, as developed for this study, will prove inadequate for providing the level of understanding needed for our students. If this occurs then the results of this study should either provide insight into a better remote learning approach, or suggest that remote learning for this application is impractical.

\section{Course Content}

The fundamental features of control system design are presented. Analysis, simulation, and hardware verification are stressed in lecture and laboratory exercises. Computer Aided Control System Design (CACSD) processes are introduced using laboratory experiments integrated with MATLAB's rapid prototyping control system design tools. Topics include: Linearity and

\author{
Proceedings of the 2004 American Society for Engineering Education \\ Annual Conference \& Exposition \\ Copyright @ 2004, American Society for Engineering Education
}


linearization, Laplace transform analysis, time domain specification based design, performance criteria: sensitivity and disturbance rejection, stability analysis and controller design via root locus and Bode plots, PID controller design and compensation.

Table 1: MEEM4700 Laboratory Syllabus

\begin{tabular}{|l|l|}
\hline Lab 1 & MATLAB Tutorial \\
\hline Lab 2 & Introduction to Diagnostic Instrumentation \\
\hline Lab 3 & Hardware/Software Introduction \\
\hline Lab 4 & Motor Cart Control - Freestyle \\
\hline Lab 5 & Motor Cart Dynamic Model Validation \\
\hline Lab 6 & Gear Train Dynamic Model Validation \\
\hline Lab 7 & Second Order System Controller Design \\
\hline Lab 8 & Root Locus Controller Design \\
\hline Lab 9 & PI Liquid-Level Controller Design \\
\hline Lab 10 & Lead Compensation using Bode Plots \\
\hline
\end{tabular}

The weekly lab lasts two hours with students working in groups of two. The course has ten labs, listed in Table 1, where five of them are two-week assignments. In most cases students are required to create a numerical simulation of the dynamic system of interest using MATLAB. After performing simulated pre-tests of the controller design, students implement it on the hardware and compare predicted to actual performance.

\section{Experiments and Equipment}

The equipment at each station can be divided into two groups: diagnostic instrumentation and control system experiments. Diagnostic instrumentation (oscilloscope, multimeter, and function generator) is available for debugging problems that may arise during the experiments. The control system experiments have three different plants: (1) a DC motor driven gear set with encoder feedback, (2) a DC motor driven cart with encoder feedback, (3) a water pump/tank system with tank pressure sensor feedback. All three systems interface with SIMULINK's realtime workshop; therefore, no low-level coding is required to implement controller designs.

The experiment modules were purchased from Quanser Consulting, including connector panels, drive electronics and PC-based input/output boards. Using Quanser supplied drivers, the experiment modules integrate with MATLAB's Real-Time Workshop and SIMULINK toolboxes. The on-campus lab requires the students to build applications in SIMULINK using the Quanser supplied I/O blocks to acquire sensor information and send actuator commands to the experiments. SIMULINK's Real-Time Workshop facilitates the automatic conversion of the student's SIMULINK block-diagram application to a real-time application that runs on the PCs at each laboratory station. Once the application is built and running successfully, students can interact with the experiment in real-time. Changing controller design parameters and reference commands are typical examples of how the student explores the effect of their designs on the closed-loop performance of the system. 
Quanser's WebLab software was used to transition the lab to a remote education application. WebLab is visualization tele-reality software that enables the user to access and control the experiments remotely. The WebLab Java bean (a reuseable component manipulated from the visual builder mode) in conjunction with the WebLab server forms the major communication link between the Matlab engine and the student's graphical user interface (GUI) via the World Wide Web. WebLab designer is used to design the GUI that the students use to interact with the experiments.

In summary, WebLab allows remote students to interact with an experiment from a web browser. A typical schematic representation is shown in Figure 1.

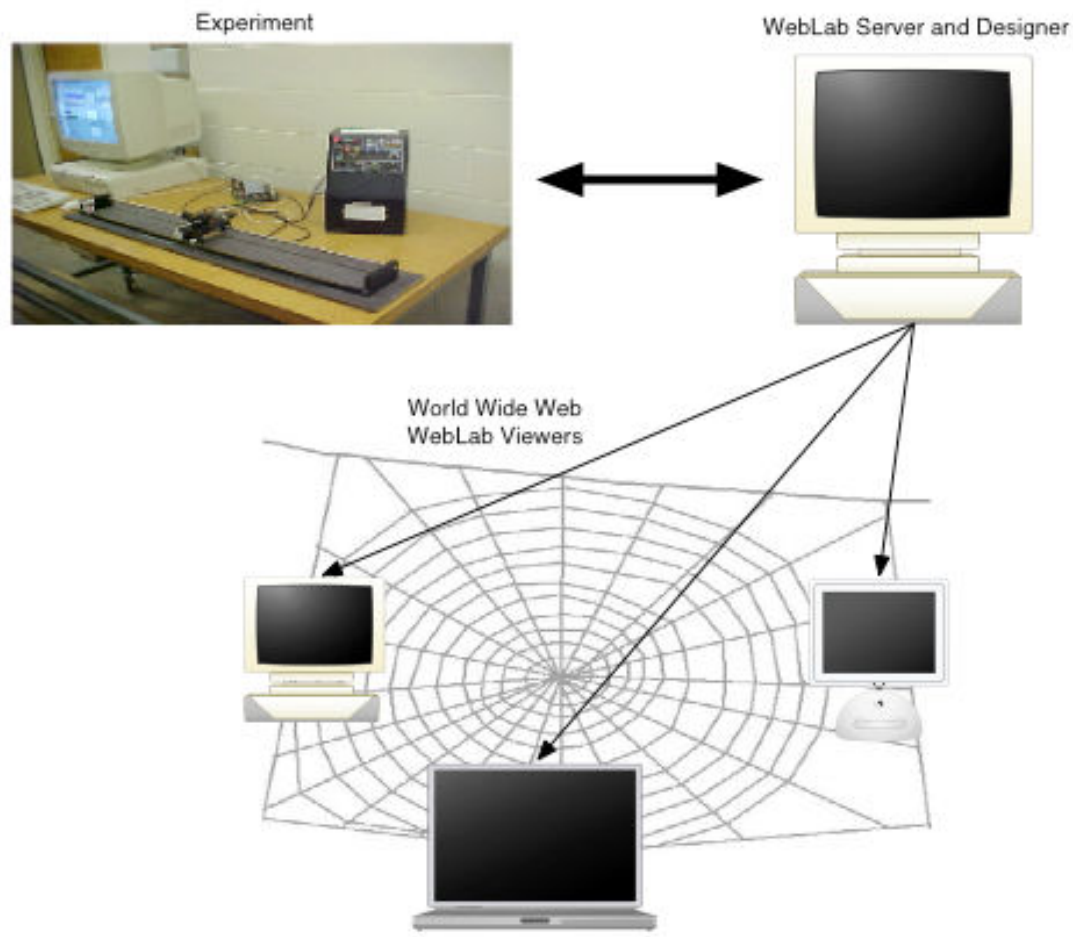

Figure 1: A single server with multiple clients, access via web or Internet

Sensor information from the remote experiment, shown in Figure 2, is streamed to the user's web application and used to render the motion of a 3-D model of the hardware (Figure 3) in near realtime. The tool provides an interface designer (Figure 4) so that Java-based web applications can be created allowing students to interact with the experiments. Control design parameters and reference commands can be changed and sensor data acquired. One limitation is that students must work from a fixed topology of the control design. Thus they do not go through the process of selecting the appropriate blocks from the SIMULINK palettes to construct the specified control architecture. A typical SIMULINK application is shown in Figure 5. One advantage to the 3-D solid model of the experiment is that the user can view the motion from any perspective. 


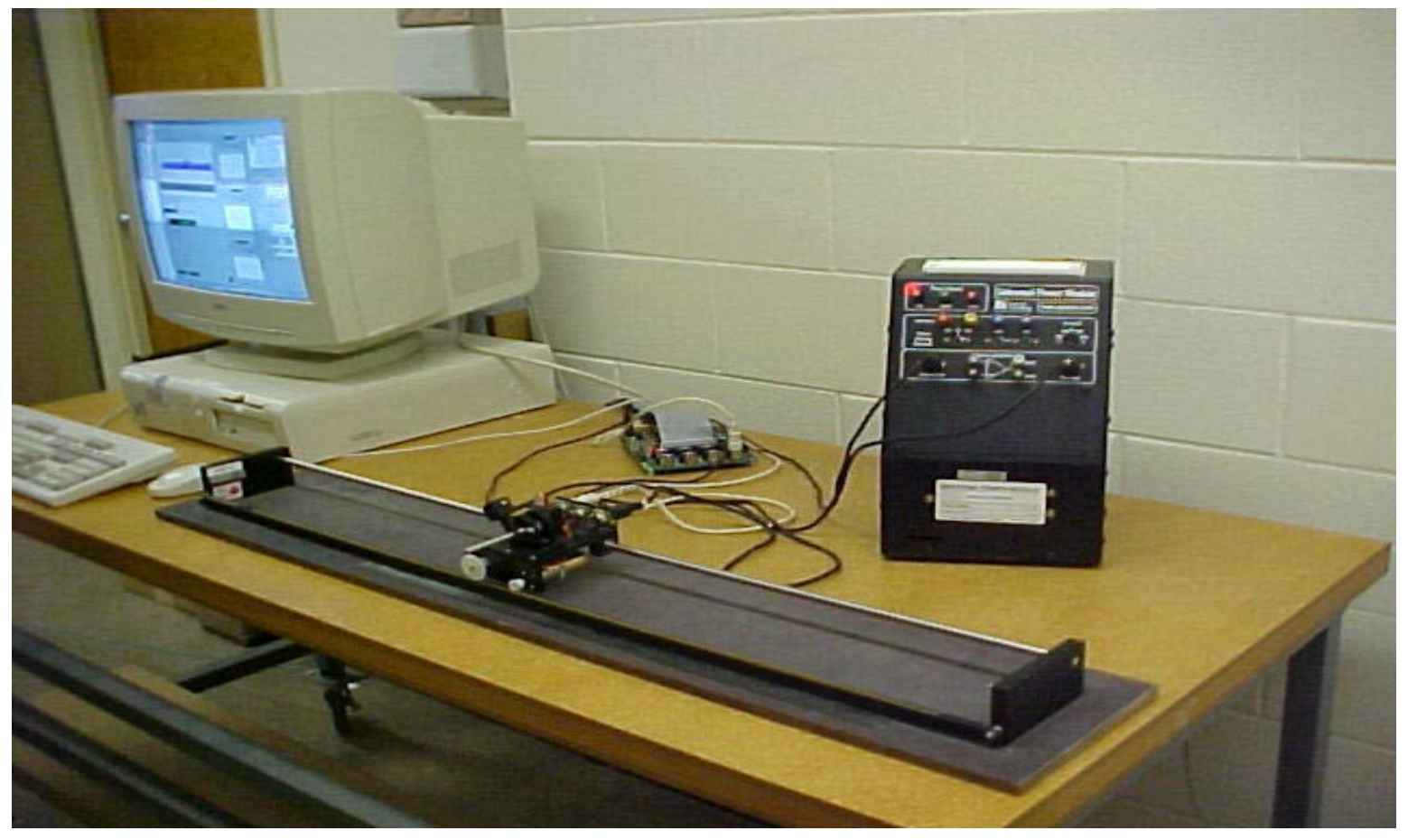

Figure 2: Hardware set-up of the DC motor driven cart/track system.

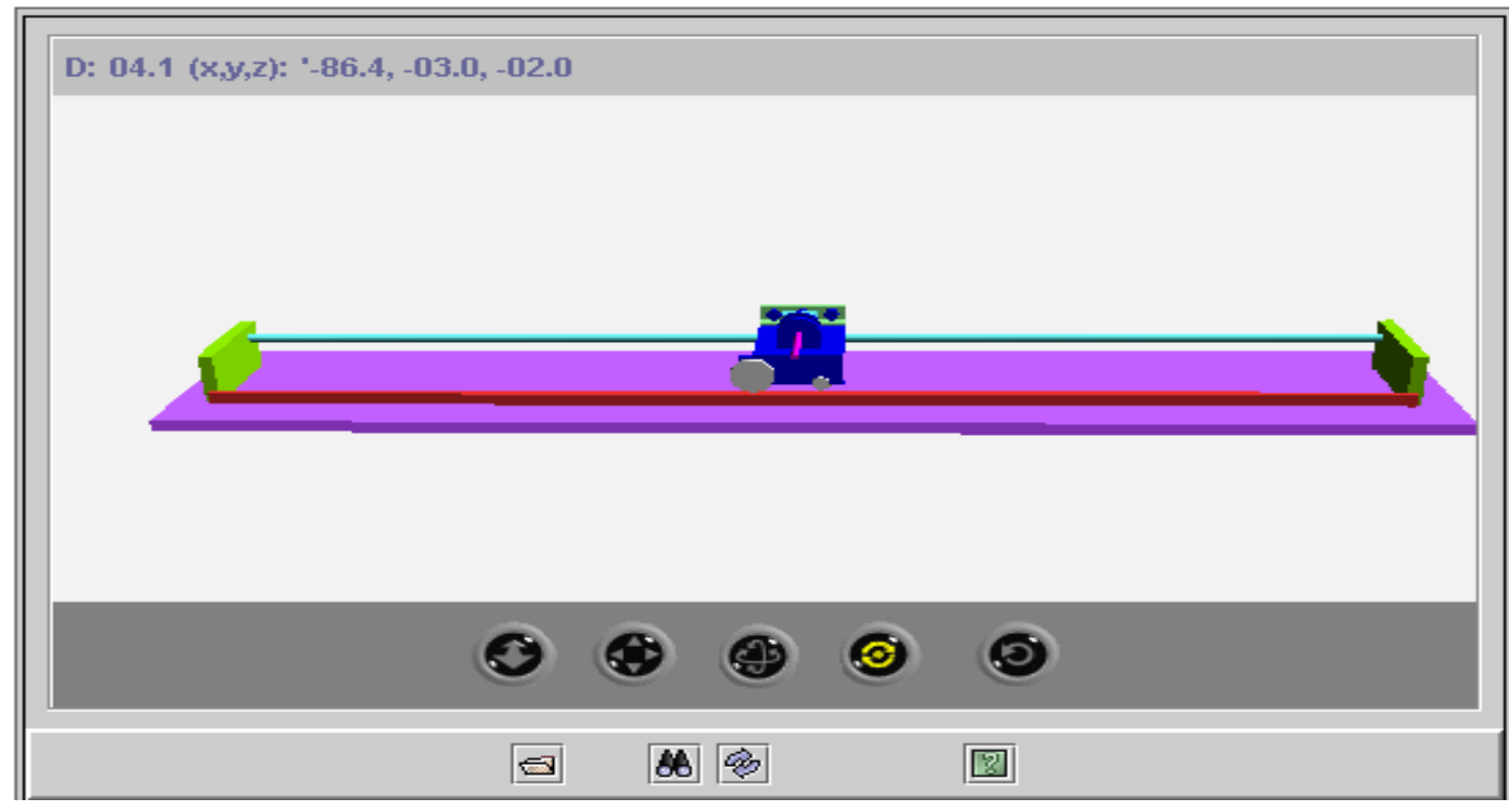

Figure 3: Close-up view of the hardware solid model of the cart/track system. 


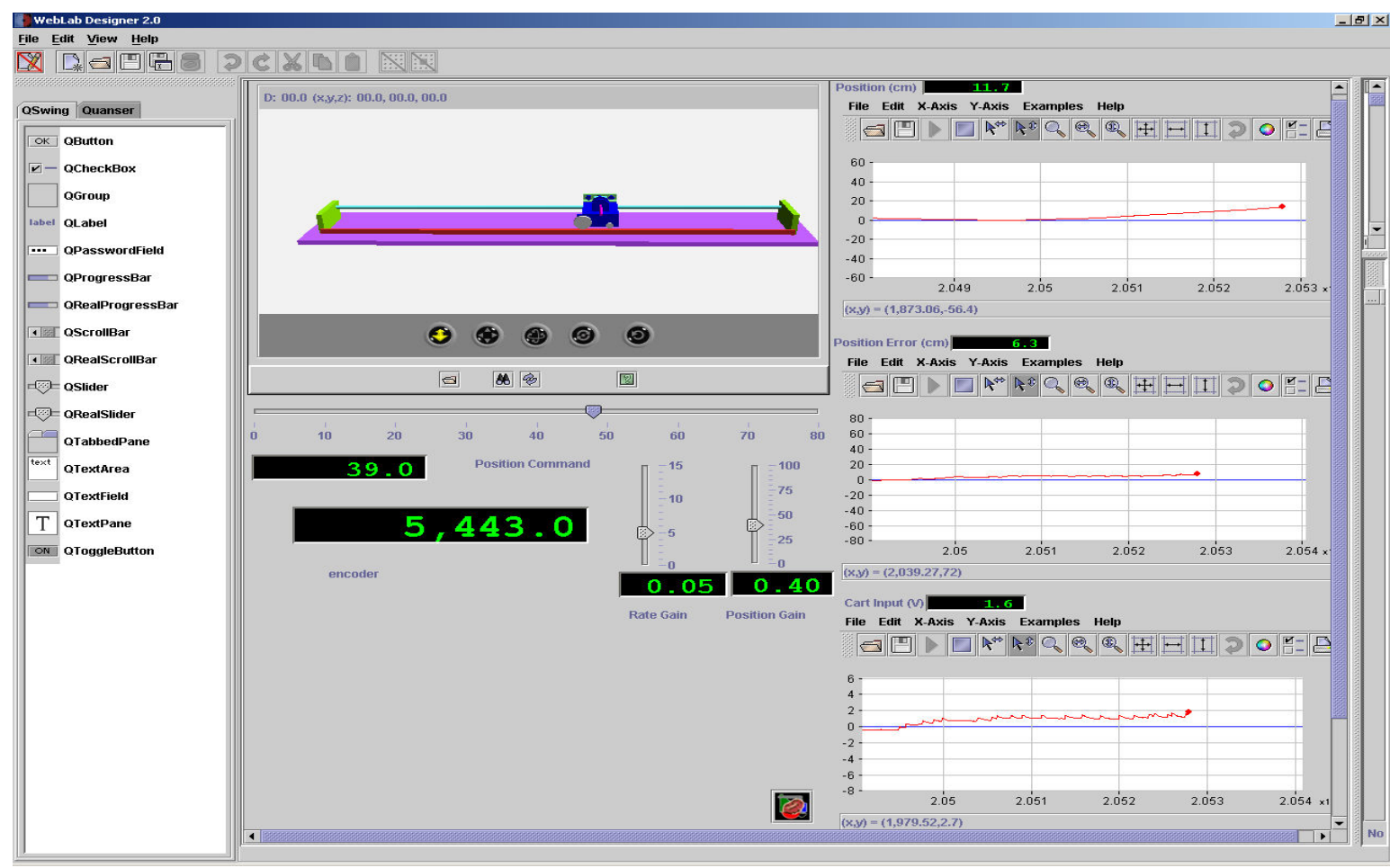

Figure 4. WebLab interface illustrating the ability to: (1) see a 3-D rendering of the remote hardware (2) manipulate control parameters (3) acquire time histories of sensor data.

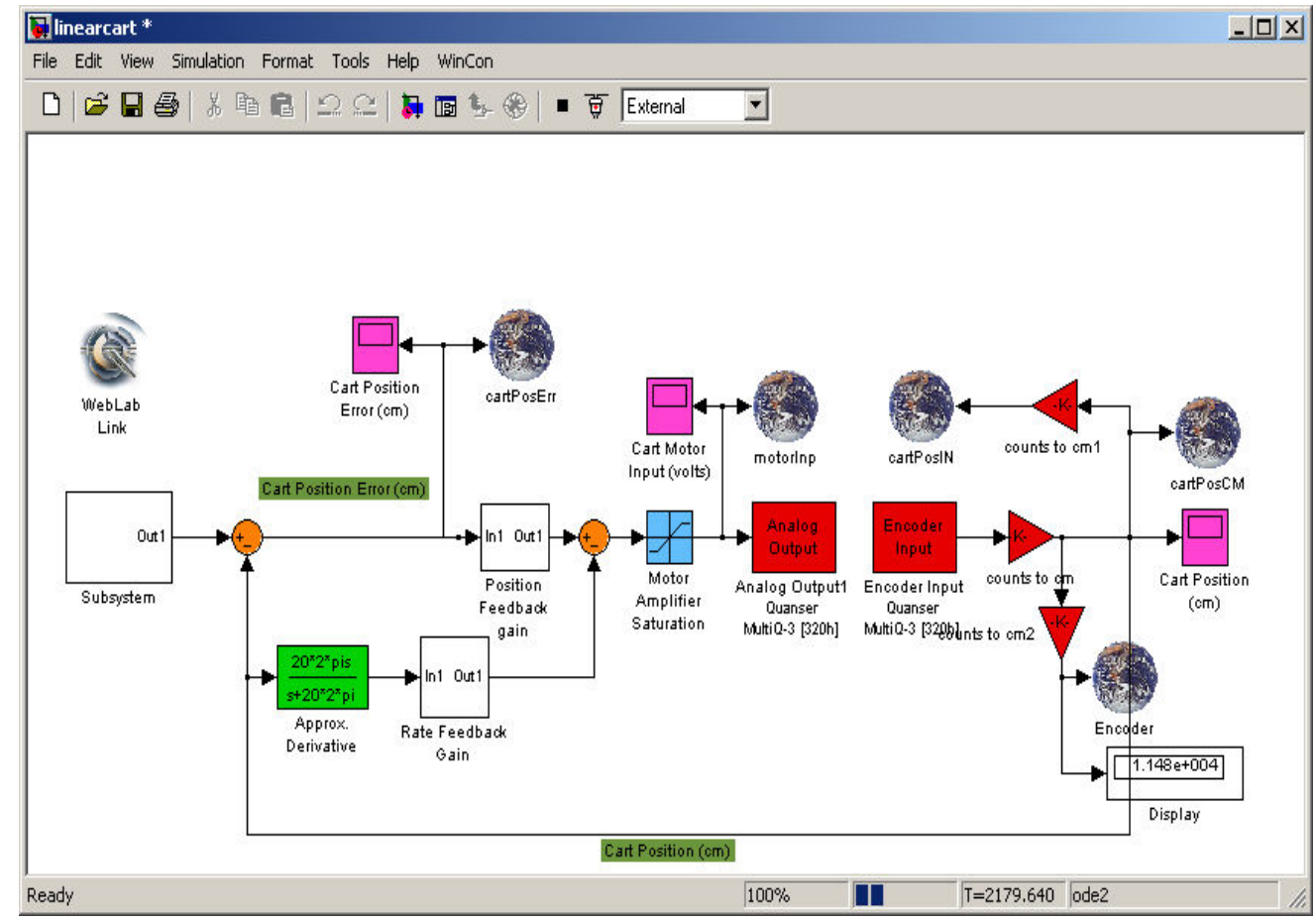

Figure 5: SIMULINK block diagram of the controller implementation. I/O points to WebLab are illustrated by the globes.

Proceedings of the 2004 American Society for Engineering Education Annual Conference \& Exposition

Copyright@ 2004, American Society for Engineering Education 


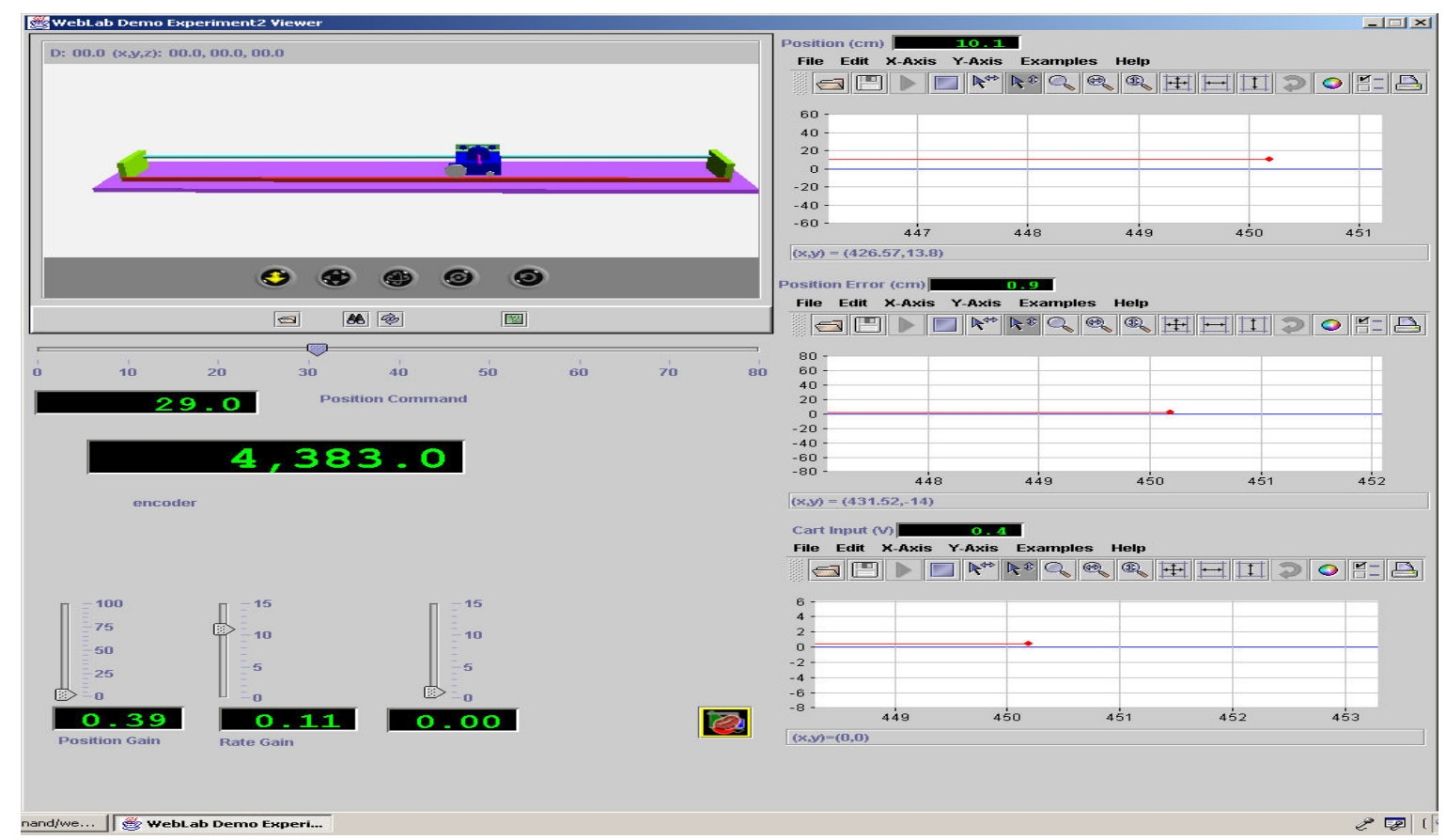

Figure 6: WebLab demo experiment viewer that can be opened by any web browser.

\section{Implementation}

The primary difference between the original, on-site laboratories and the remote learning labs is that the WebLab applications require the control strategy topology to be made available to the students. Our concern was that the lab experience would be lessened if the students did not have the experience of building the SIMULINK control application from the basic building blocks provided by MATLAB. To overcome this potential problem 5 of the 10 labs were constructed as two-week activities. In the first week students design a simulation of the experiment with a specified control strategy. During this exercise they go through the experience of building the low-level SIMULINK control strategy and quantify the theoretical response of their closed-loop system. In the second week they are presented with the WebLab version of the same control strategy, but, it is driving the remotely located hardware experiment. This phase of the lab allows the students to not only explore different control designs through gain selection, but also to compare their theoretical results with those of the hardware.

\section{Planned Assessment}

The Department of Mechanical Engineering - Engineering Mechanics at Michigan Tech has a large undergraduate population, with approximately 250 seniors. The Dynamic Systems and Controls course is a required senior-level course and is offered every semester. The plan is to have several sections of students conduct the labs as though they were remote. That is, they would not have direct Teaching Assistant contact and would perform the labs over the web.

Two different tools will be used to measure the student's learning of laboratory concepts. First, as mentioned above, a student's laboratory success is measured through questions on all the

Proceedings of the 2004 American Society for Engineering Education Annual Conference \& Exposition

Copyright@ 2004, American Society for Engineering Education 
course exams. Approximately $15 \%$ of each exam has questions dedicated to laboratory content. Differences between the on-site and "virtual" remote students can be easily tabulated from these results. The second tool will be a separate exam that will not count towards the student's actual grade. This will ask questions focused on concepts that are typically only learned through handson exploration of an experiment. A typical example would be to determine if a student could describe what it feels like when they push on the cart while it is under closed-loop, proportional control. That is, can they identify that it feels like a spring pushing back on their hand. Most students quickly learn this when using the hardware in the laboratory. Learning this through the 3-D model, remote version may be difficult. Another example is to determine if students understand how external disturbance affect the closed-loop performance of the system. Again, in lab students are asked to apply external disturbances with their hands, or by lifting the base of the equipment. It will be interesting to see if the remote students acquire the same level of understanding of the system performance.

\title{
Conclusions
}

It is unlikely that a web-based application will provide students with the same level of interaction as a hands-on laboratory. However, some learning objectives may be handled remotely, and for these, a web-based system may be appropriate. The work described in this paper is part of an effort to understand the current capability of a particular web-based remote learning tool and to eventually understand the comprehension differences between hands-on and remote lab instruction. Preliminary testing of a WebLab experiment has been completed using undergraduate student volunteers. The primary goal was to investigate how students would interact with the interface and obtain suggestions for performing a complete course, remote lab test in Fall 2004. The students learned the interface quickly, and were able to collect and analyze data as well as test different closed-loop control strategies successfully. One suggestion that will be implemented for the full test is to stream video of the actual hardware. Another enhancement that will be explored is to have real-time communication with a TA in the room where the hardware experiments are being run. This will provide instantaneous feedback to student questions with guidance how to explore their designs further.

\section{Acknowledgements}

The Departments of Mechanical Engineering - Engineering Mechanics and Sponsored Educational Programs at Michigan Technological University, supported this work. The authors would like to thank Andrew Richards, Michael Svendsen and Jose Cabrera for their help in testing the WebLab application as part of their Dynamic Systems and Controls course. Software technical support from Vincent Lo and Andrew Dawes at Quanser Consulting and computer support from Michigan Tech's Javier Fernandez and Matt Heyse was appreciated and essential for creating a useful interface under the time constraints required by the study.

GORDON G. PARKER

Gordon Parker is an Associate Professor in the Mechanical Engineering Department at Michigan Tech where his research interest is control systems. He received the Ph.D. degree in mechanical engineering from State University

\author{
Proceedings of the 2004 American Society for Engineering Education \\ Annual Conference \& Exposition \\ Copyright@ 2004, American Society for Engineering Education
}


of New York at Buffalo in 1994. From 1989 to 1996, he worked at General Dynamics, Space Systems and Sandia National Laboratories in the areas of structural dynamics and control.

MICHAEL J. AGOSTINI

Michael Agostini is a Ph.D. candidate in the Mechanical Engineering Department at the Michigan Technological University where he received both his M.S. and B.S. His research interests include nonlinear control and adaptive systems.

\section{MARUTHI DEVARAKONDA}

Maruthi N. Devarakonda is a Ph.D student in the Mechanical Engineering Department at the Michigan

Technological University. He received a B.S degree in Mechanical Engineering from Nagarjuna University, India in June 2000 and an M.S degree in Mechanical Engineering from University of Toledo in August 2003. His research interests include input shaping and time optimal solutions for nonlinear systems.

PAULA F. ZENNER

Paula Feira Zenner is the Director of Operations and Finance for the Department of Mechanical EngineeringEngineering Mechanics at Michigan Technological University. She received a B.S. degree in Mechanical Engineering from Michigan Tech in 1987 and an M.S. degree in Operations Management from Michigan Tech in 1993. Before returning to graduate school she spent four years as a Technical Specialist in the computer industry. 